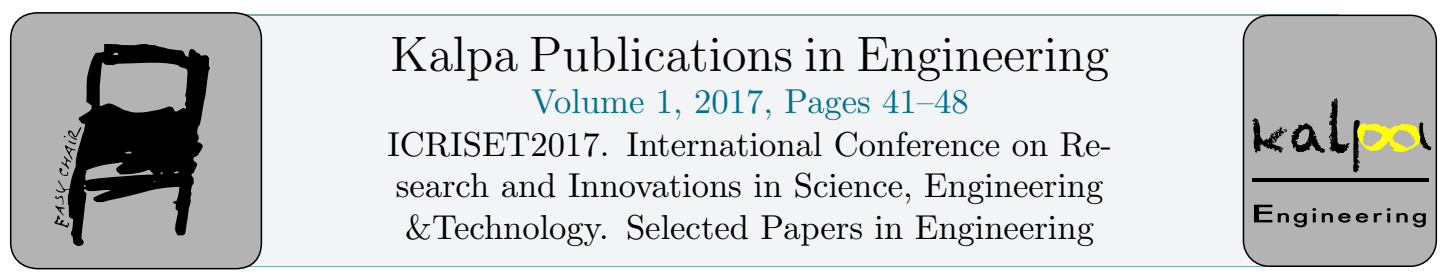

\title{
Design and Development of On-Board Earth Resources Detection System Based on FPGA and LabVIEW for Remote Sensing Application
}

\author{
Alpesh Vala ${ }^{1}$, Riddhi Goswami ${ }^{2}$, Amit Patel, Keyur Mahant \\ CHARUSAT Space Research \& Technology center \\ Charotar University of Science \& Technology, Changa, Anand, Gujarat-388421 \\ alpeshvala.ec@charusat.ac.in, riddhigosai 8881 @gmail.com
}

\begin{abstract}
This paper presents system development for the detection of earth resources such as a water, vegetation and land for the satellite application. Satellite imaging sensors generate mass volume of data at very high speeds. On the other hand, storage capacity and communication bandwidth are crucial parameters for satellite resources. Here we have proposed the system that can be used on board to extract relative information from the image and can send out the required (obtained) results to the ground system (Result of pixel information whether it contains water/vegetation/land). The system can be used for the saving of on board satellite resources such as memory storage, power and communication bandwidth. The detection of earth resources are based on their reflectance value. For the analysis of proposed detection algorithm LabVIEW based simulation has been carried out for the detection of Land, Water and vegetation from their reflectance value. Same algorithm has implemented in FPGA for the real time implementation using SPARTAN XC3S500e-4vq100 FPGA board. The results are accurate and matched with the simulation results performed in LabVIEW.
\end{abstract}

Keywords: Land, Water, Vegetation, Field Programmable Gate Array (FPGA), LabVIEW ( Laboratory Virtual Instrument Engineering Workbench)

\section{Introduction}

Remote sensing is being used for the detection and classification of objects on earth such as land, vegetation, water, sand, dessert, snow. This information can be used for the mapping land use, archaeological investigation, military observation, agriculture, soil mapping, city planning, forestry, geographical surveying etc. [1]. 
These operations are generally performed by satellite in store and forward modes. It means first image is captured by satellite camera (sensors), stored that image in onboard memory and send the resultant data to the ground subsystem with the use of downlink channel[1].This process utilizes onboard satellite resources such as memory and communication bandwidth for the transmission of resultant data. To utilize better resources of satellite, it is better to use on-board system instead of store and forward mode. In which processing of required data is done on satellite and send out the resultant data to the ground station (for better utilization of channel bandwidth).

To detect geophysical object such as vegetation, water, land, snow, cloud at onboard, it is important that it should operate with high computational accuracy [2]. In modern satellite remote sensing technology an algorithm implementation to detect these geophysical objects is carried out at the ground subsystem level [1]. Ordinary methods to detect these object based on individual or combination of method such as spectral contrast, radiance temporal contrast, and spatial contrast. A motivation behind implementation of algorithm for detection of geophysical object on hardware is that it can be used on onboard to reduce transmission of inconsequential image data to the ground subsystem.

There are number of possibilities to implement the algorithm on hardware such as DSP, FPGA, Microcontroller, Processor etc. FPGA has a unique advantage such as reconfigurable logic cells, ability to perform logical operation according to user application, ability to handle parallel operation. It can potentially reduce the delay between image capture, analysis and action. It also reduces onboard storage and downlink capacity requirements [3].

The flow of paper is as follows. Section II describes the related work that has been previously done for the detection of land, water and vegetation detection based on reflection. Section III describes algorithm outline for the detection of water, vegetation and land and simulation results using LabVIEW for the analysis and verification purpose. Section IV describes the hardware description and result of algorithm implementation in hardware.

\section{Literature Survey}

The detection of earth resources have wide range of applications such as an environmental monitoring, climate changes studies, numerical weather forecast model, natural hazard monitoring, agriculture and forestry [4]. A Basic concept related to the reflectance quantities are classified in literature [5]. Reflectance term such as Bidirectional reflectance distribution function (BRDF), Hemispherical-directional reflectance factor (HDRF), Directional-hemispherical reflectance (DHR), Bi hemispherical reflectance (BHR), albedo are explained in this literature with details. Review of water body extraction using satellite remote sensing is presents in literature [6]. A tree species classification from high resolution colour infrared aerial images and its digital surface model is described in literature [7]. The identification strategy for the tree species includes detection of vegetation area, segmentation of vegetation area according to their height followed by identification of individual tree.

To find out the spectral signature of objects like land cover classification and the vegetation index on low contrast satellite images using normalise difference vegetation index technique is present in literature [8]. Singular value decomposition and discrete cosine transformation techniques are used for the features extraction on low contrast satellites images. Vegetation detection algorithm for the close range images based on texture features and naive bayes classifier is presented in literature [9].To prove the validity of the method they have compare the method with the visual cognition features. To eliminate vegetation's interference with the landslide deformation monitoring the result of vegetation detection is applied to landslide monitoring [9]. Huge amount of studies are performed on satellite and aerial images for the detection of vegetation [9-11]. 
It is mentioned in [12] that both NDVI (Normalized Difference Vegetation Index) and NDWI (Normalized Difference Water Index) method do not use more than two bands to calculate index which misrecognize some non-water features as their index values fall close to index values of water features. Hence, Hongxu has defined water detection method which is combination of three methods NDWI, NDVI and mathematical morphology [12]. Min Li has proposed method that uses locally excitatory globally inhibitory oscillator networks which can extract coherent water body without the analysis of water and its surround environment [13]. Hanqiu Xu has proposed modified NDWI algorithm which can discriminate open water features from non-water features while efficiently suppressing and even removing built-up land noise as well as vegetation and soil noise [14].

\section{Algorithm Outline and Simulation Results}

Fundamental source of energy is the sun and it radiates the electromagnetic energy in earth atmosphere. Some of this energy absorbed in earth surface and some are reflected back. It is possible to detect earth resources from its reflectance value. Reflectance value of any object can determined from the albedo (reflectance radiation from earth surface). This albedo can be figure out by taking ratio of reflectance to the total radiation hitting to the surface. Figure 1 shows the reflectance value of different object from the earth surface. Additional detail of reflectance value is tabulated in Table I.

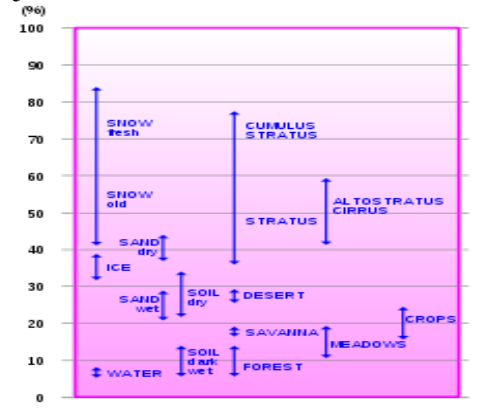

Figure 1: Reflectance value of different object from earth surface

\begin{tabular}{|c|c|}
\hline Surface & Reflectance (\%) \\
\hline Soil & $5-40$ \\
\hline Grass & $16-26$ \\
\hline Forest & $15-25$ \\
\hline Water & $3-10$ \\
\hline Snow & $40-95$ \\
\hline Ice & $30-50$ \\
\hline
\end{tabular}

Table 1: Reflectance Value of Different Object Relative to Earth Surface

\subsection{Algorithm Development and Analysis}

With the analysis of different images in LabVIEW, we have derived reflectance range of red, green and blue components of individual pixel for land, water and vegetation. We have found that for detecting vegetation red component of individual pixel varies in range of 25 to 50 , green component of individual pixel varies in range of 20 to 60; blue component of individual pixel varies in range of 25 to 45 . Same way for water the pixel has value of red component in between 30 to 60 , value of green component in between 35 to 60, and Value of blue component in between 30 to 90 . For the land red component varies in range of 90 to 110 , green component varies in range 80 to 110; blue component varies in range of 60 to 110.Further to this, we have derived the ratio of red to green component and ratio of blue to green components of individual pixels for the individual objects. The reflectance range and ratio of red/green and blue/ red are tabulated in the table 2 . The complete flow of the algorithm for the detection of earth resources is shown in figure 2. 


\begin{tabular}{|c|c|c|c|c|c|c|c|c|}
\hline \multirow{2}{*}{ Surface } & \multicolumn{6}{|c|}{ Reflectance } & \multicolumn{2}{c|}{ Ratio } \\
\cline { 2 - 6 } & \multicolumn{2}{|c|}{ Minimum } & \multicolumn{2}{c|}{ Maximum } & \multirow{2}{*}{ R/G } & B/R \\
\cline { 2 - 6 } & R & G & B & R & G & B & & \\
\hline Land & 90 & 80 & 60 & 110 & 110 & 110 & $\begin{array}{c}1<=\mathrm{R} / \mathrm{G} \\
<1.15\end{array}$ & $\begin{array}{c}<= \\
1\end{array}$ \\
\hline Water & 30 & 35 & 30 & 60 & 60 & 90 & $\begin{array}{c}0.8<\mathrm{R} / \mathrm{G} \\
>=1\end{array}$ & $\begin{array}{c}>= \\
1\end{array}$ \\
\hline Vegetation & 25 & 20 & 25 & 50 & 60 & 45 & $\begin{array}{c}0.8<\mathrm{R} / \mathrm{G} \\
<1.3\end{array}$ & $\begin{array}{c}<= \\
1\end{array}$ \\
\hline
\end{tabular}

Table 2:Analysis of various Ranges of values for detection of land, water and vegetation

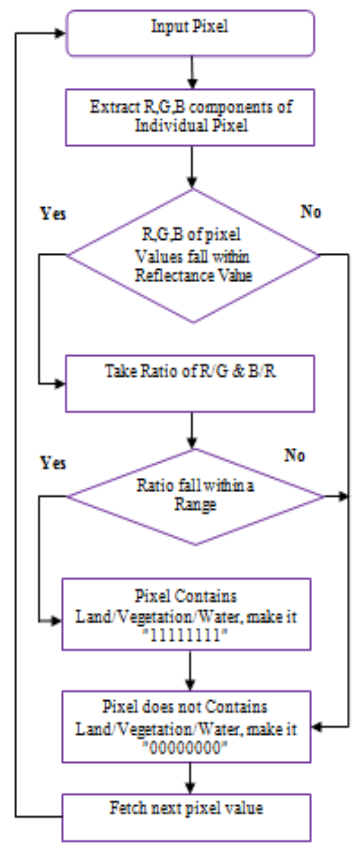

Figure 2: Flow diagram of algorithm implementation

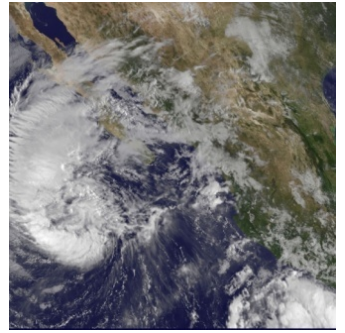

(a)

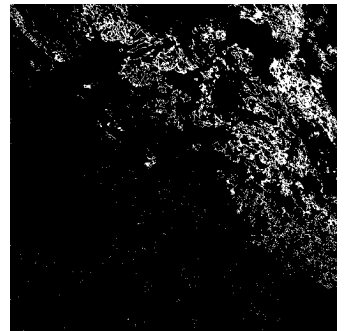

(b)

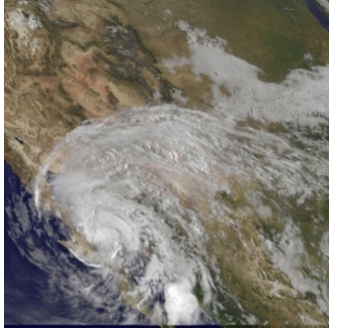

(c)

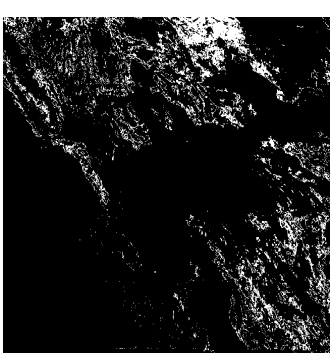

(D)

(a) 32 bit image of NOAA's GOES satellite $(512 * 512$ pixels) (b) Simulation result of figure (a) in Lab VIEW (8 bit image of 512*512 pixels) for land detection (white spot indicates presents of Land in the IMAGE) (c) 32 bit image of NOAA's GOES satellite (512* 512 pixels) (d) Simulation result of figure (c) in Lab VIEW (8 bit image of $512 * 512$ pixels) for land detection (white spot indicates presents of Land in the IMAGE)

Figure 3 : Simulation Results of Land detection in LabVIEW 
Simulation results for the detection of land, water and vegetation detection is shown in figure 3 ,figure 4 and figure 5 respectively . LabVIEW is used for the simulation purpose. First we have extracted value of Red, green, and blue components from the individual pixels. Based upon this we have decided the reflectance range value of R,G,B component of individual pixels. Following to this step the ratio of red component to the green component and ratio of blue component to the red component of individual pixel are taken.

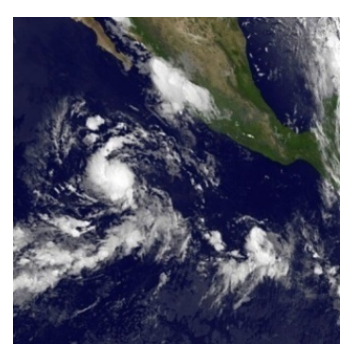

(a)

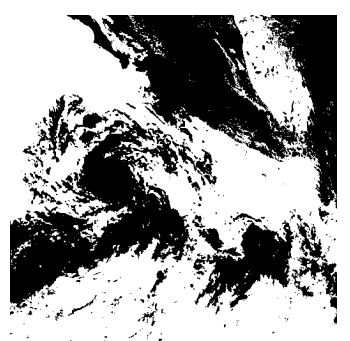

(b)

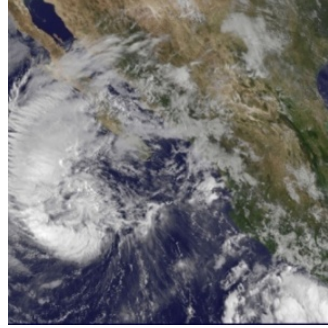

(c)

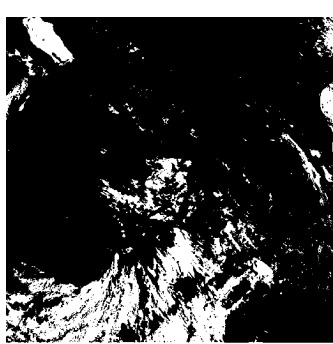

(D)

(a) 32 bit image of NOAA's GOES satellite $(512 * 512$ pixels) (b) Simulation result of figure (a) in Lab VIEW (8 bit image of $512 * 512$ pixels) for water detection (white spot indicates presents of water in the IMAGE) (c) 32 bit image of NOAA's GOES satellite $(512 * 512$ pixels) (d) Simulation result of figure (c) in Lab VIEW (8 bit image of $512 * 512$ pixels) for water detection (white spot indicates presents of water in the IMAGE)

Figure 4: Simulation Results of Water detection in LabVIEW

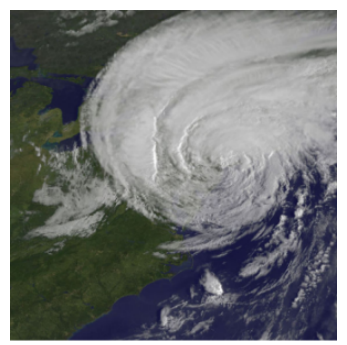

(a)

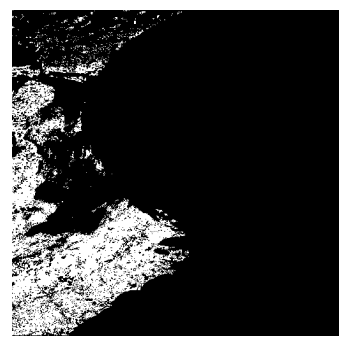

(b)

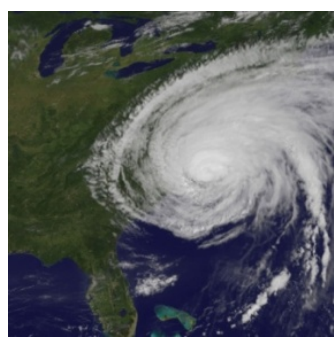

(c)

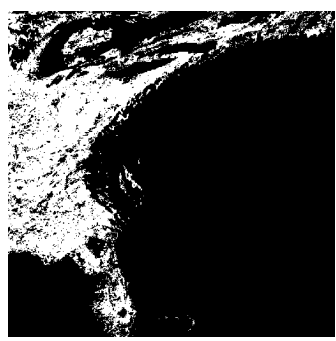

(D)

(a) 32 bit image of NOAA's GOES satellite $(512 * 512$ pixels) (b) Simulation result of figure (a) in Lab VIEW (8 bit image of $512 * 512$ pixels) for vegetation detection (white spot indicates presents of vegetation in the IMAGE) (c) 32 bit image of NOAA's GOES satellite $(512 * 512$ pixels) (d) Simulation result of figure (c) in Lab VIEW (8 bit image of $512 * 512$ pixels) for vegetation detection (white spot indicates presents of vegetation in the IMAGE)

Figure 5: Simulation Results of Water detection in LabVIEW

\section{Hardware Implementation}

We have designed and developed external board that will work as a daughter board of FPGA. It contains 2 SRAM (for the storage purpose with the configuration of $2 \mathrm{M} \times 16$ ), interface section to connect board with sensor (for future use) and for the connection of system with other system (for the data transmission purpose). KETHLEY 2230-30-1 regulated D.C. power supply is used to deliver supply voltage to FPGA and SRAM. NI LABVIEW 9 is used for the acquisition of an image and exhibit the end results. RS232 serial communication is used for the communication between FPGA board and LABVIEW. A basic block diagram of hardware implementation is shown in figure 6. An image of $512 \times 512$ pixels of 32 bit resolution is first sent from LABVIEW to FPGA using RS232 serial communication at the speed of $500 \mathrm{KBPS}$. FPGA has only $360 \mathrm{~K}$ on chip internal memory, so 
first image is stored into external SRAM ( access time of SRAM is $65 \mathrm{~ns}$ ). To extract R, G and B component of individual pixel, pixel by pixel read operation is performed. The implementation steps for the detection of earth resources are same that is described as flowchart in figure 7. The results of the pixel are transmitted to LabVIEW for the display purpose using RS232 serial communication at a speed of $500 \mathrm{KBPS}$.

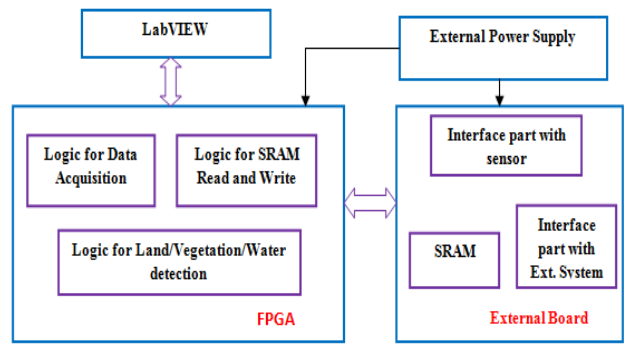

Figure 6: Basic Block of Hardware Implementation

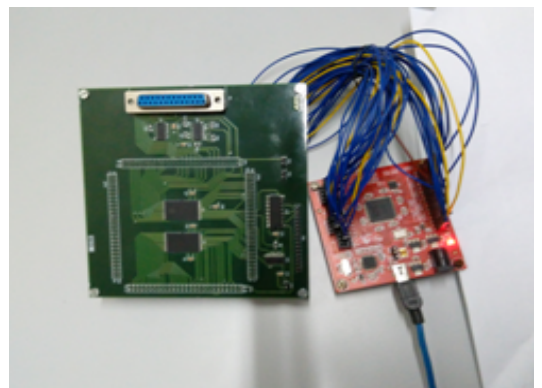

Figure 7: Hardware Setup for System implementation

The result of an algorithm implementation for the land, water and vegetation detection in hardware is shown in figure 8 ,figure 9 and in figure10 respectively.

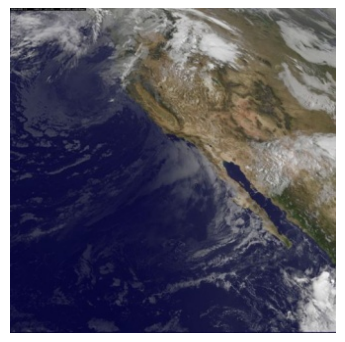

(a)

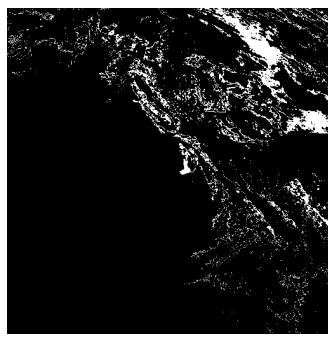

(b)

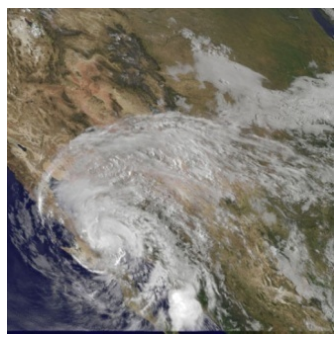

(c)

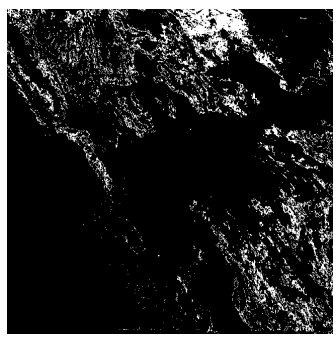

(D)

(a) 32 bit image of NOAA's GOES satellite $(512 * 512$ pixels) (b) Results in Hardware of figure b (8 bit image of $512 * 512$ pixels) for land detection (white spot indicates presents of Land in the IMAGE) (c) 32 bit image of NOAA's GOES satellite (512* 512 pixels) (d) Results in Hardware of figure c ( 8 bit image of $512 * 512$ pixels) for land detection (white spot indicates presents of Land in the IMAGE)

Figure 8: Results of algorithm implementation in hardware for land detection

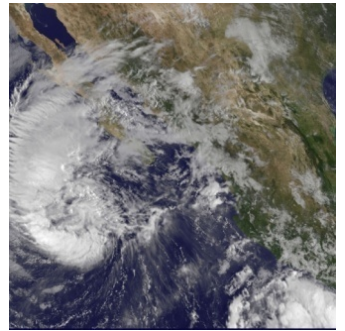

(a)

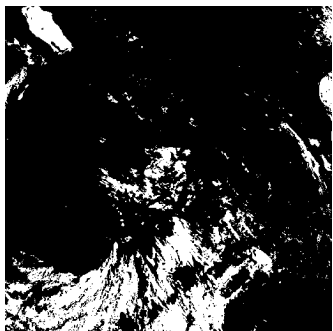

(b)

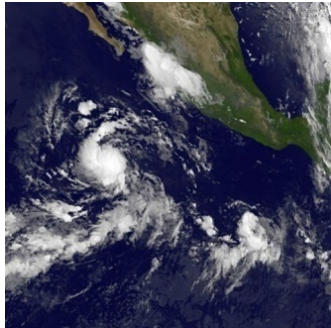

(c)

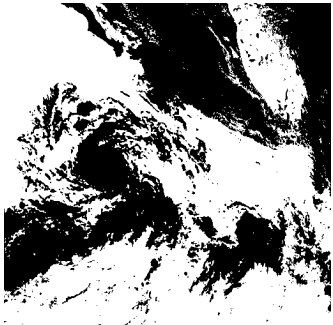

(D)

(a) 32 bit image of NOAA's GOES satellite $(512 * 512$ pixels) of Baja California (b) Results in Hardware (8 bit image of $512 * 512$ pixels) for water detection (c) 32 bit image of NOAA's GOES satellite (512*512 pixels) (d) Results in Hardware ( 8 bit image of $512 * 512$ pixels) water detection

Figure 9: Results of algorithm implementation in hardware for water detection 


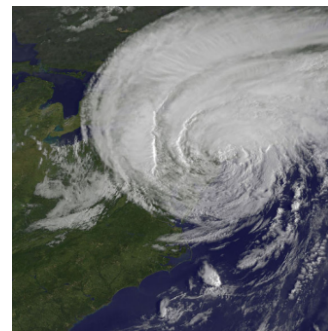

(a)

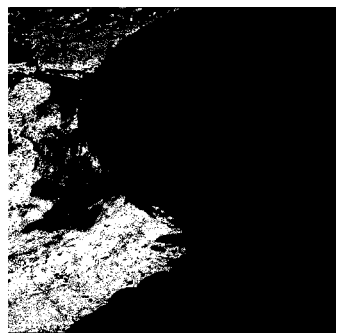

(b)

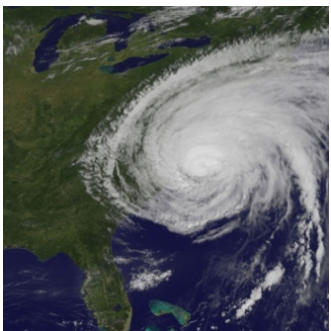

(c)

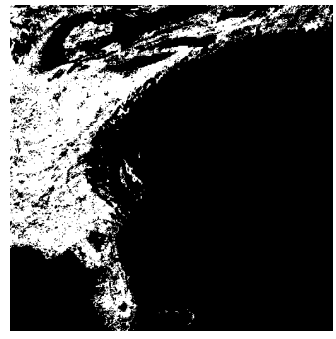

(D)

(a) 32 bit image of NOAA's GOES satellite ( $512 * 512$ pixels) (b) Results in Hardware ( 8 bit image of $512 * 512$ pixels) for vegetation detection (c) 32 bit image of NOAA's GOES satellite $(512 * 512$ pixels) (d) Results in Hardware (8 bit image of $512 * 512$ pixels) vegetation detection

Figure 10: Results of algorithm implementation in hardware for vegetation detection

\subsection{Result and Analysis}

For the comparison purpose, we have compared the results implemented in hardware with the simulation results of LabVIEW, It shows more than 90 percents of results are matched with the simulation results. Device utilization summary of FPGA is tabulated in table 3.

\begin{tabular}{|c|c|c|c|}
\hline Logic Utilization & Used & Available & $\begin{array}{c}\text { Utilization } \\
\text { (Percentage) }\end{array}$ \\
\hline Number of Slice flip flops & 438 & 9312 & 4 \\
\hline Number of 4 input LUTs & 521 & 9312 & 5 \\
\hline Number of occupied slices & 473 & 4656 & 10 \\
\hline Total number of 4 input LUTs & 845 & 9312 & 9 \\
\hline Number of bonded IOBs & 47 & 66 & 71 \\
\hline Number of BUFGMUXs & 3 & 24 & 12 \\
\hline Frequency of operation & \multicolumn{3}{|c|}{$32 \mathrm{MHz}$} \\
\hline
\end{tabular}

Table 3:Utilization of FPGA resources

\section{Conclusion}

A detection of earth resources such as land, vegetation and water are simulated and implemented in the hardware based on its reflectance value. LabVIEW is used for the simulation purpose and hardware implementation is carried out in FPGA. From the results, it is cleared that the proposed algorithm performs very well for detection of land, water and vegetation. All the simulation results of LabVIEW are compared with FPGA results shows the more than $90 \%$ accuracy of algorithm. The utilization of resources on hardware with this algorithm is very less and also the total power consumption of the system is around $400 \mathrm{~mW}$. It is further possible to detect other earth resources such as cloud, snow, sand etc with the use of same algorithm according to reflectance values of objects. It is possible to use the proposed system on-board (satellite) with the optic sensor configuration.

\section{References}

[1] Esam El-Araby, Tarek El-Ghazawi, Jacqueline Le Moigne, Richard Irish "Reconfigurable processing for satellite onboard automatic cloud cover assessment" Journal of Real time Image Processing, Vol.4,2009. 
[2] Ashok N. Srivastava, Julienne Stroeve " Onboard detection of snow, ice, clouds and other geophysical processes using kernel methods" Proceedings of the ICML 2003Workshop on Machine Learning Technologies for Autonomous Space Sciences

[3] John A. Williams, Anwar S. Dawood and Stephen J. Visser "FPGA-based cloud detection for real-time onboard remote sensing" IEEE International Conference on Field-Programmable Technology (FPT),2002

[4] Corina Iovan, Didier Boldo And Matthieu Cord "Detection, segmentation and characterisation of vegetation in highresolution aerial images for 3d city modelling" The International Archives of the Photogrammetric, Remote Sensing and Spatial Information Sciences. Vol. XXXVII. Part B3a. Beijing 2008.

[5] G. Schaepman-Strub, M.E. Schaepman, T.H. Painter, S. Dangel, J.V. Martonchik " Reflectance quantities in optical remote sensing - definitions and case studies" Remote Sensing of Environment, Vol 103, Issue 1, 15 July 2006

[6] Rajiv Kumar Nath, S K Deb "Water-body area extraction from high resolution satellite images-an introduction, review, and comparison" International Journal of Image Processing, Volume 3, Issue 6, February 2010

[7] Corina Iovan,Didier Boldo ,Matthieu Cord "Detection, segmentation and characterisation of vegetation in high-resolution aerial images for $3 \mathrm{~d}$ city modelling" The International Archives of the Photogrammetry, Remote Sensing and Spatial Information Sciences, Vol. XXXVII. Part B3a. Beijing 2008

[8] A. Kumar, A.K Bhandri, P. Padhy " Improved normalised difference vegetation index method based on discrete cosine transorm and singular value decomposition for satellite image processing" IET Journal of Image Processing, Volume 6, September 2012

[9] Zongqian Zhan, Binghua Lai " Vegetation detection of close-range images for landslide monitoring" International Conference on Computer Vision in Remote Sensing (CVRS), December 2012

[10] J.H. Liu. "Study on Visual Recognition and Automatic Extraction of Typical Ground Objects in Remote Sensed Images". Ph.D. dissertation, Department of Automation Shanghai Jiao Tong University, 2010.

[11] Li. "Research on Vegetation Detection Algorithm of Close-range Images". Bachelor thesis, Wuhan University, 2010.

[12] Hongxu Ma, Shenglian Guo and Yanlai Zhou "Detection of water area change based on remote sensing images"GeoInformatics in Resource Management and Sustainable Ecosystem, Vol.398 of the series communications in computer and iformationscience.

[13] Min Li , Lizhong Xu and Min Tang,"An extraction method for water body of remote sensing image based on oscillatory network "Journal of multimedia, Vol.6,No.3,June2011

[14] Hanqiu Xu , "Modification of normalised difference water index(NDWI) to enhance open water features in remotely sensed imagery" International Journal of Remote Sensing, Vol.27,No.14,20 July2006,3025-3033. 